\title{
El constructivismo sociocultural lingüístico como teoría pedagógica de soporte para los Estudios Generales
}

Linguistic Sociocultural Constructivism as a Founding Pedagogical Theory for General Studies

\author{
María Elena Córdoba \\ Instituto Tecnológico de Santo Domingo
}

Argentina

\section{Resumen}

El presente artículo tiene como objetivo, señalar y analizar algunas características del constructivismo y de los Estudios Generales con intención de establecer puentes conceptuales y de encuentro metodológico entre ambos, así como generar la reflexión que permita identificar, específicamente, las bondades del constructivismo sociocultural lingüístico como un soporte teórico adecuado a la epistemología de los Estudios Generales, cuyo propósito se orienta al desarrollo de ciudadanos éticos, con pensamiento crítico y sentido del bien común, entre otras características. La relación de horizontalidad, que proponen los Estudios Generales, y el vínculo docente-estudiante, donde me reconozco y reconozco al otro como persona, no pueden estar sostenidos por cualquier teoría pedagógica. Por tal razón, es posible empezar a establecer lazos con esta modalidad de constructivismo, en la cual el proceso de enseñanza y aprendizaje implica actividad conjunta entre estudiantes y docentes, y acontece alrededor de contenidos y acciones de enseñanza a través de la estructuración de esa actividad y de los recursos semióticos presentes en el habla

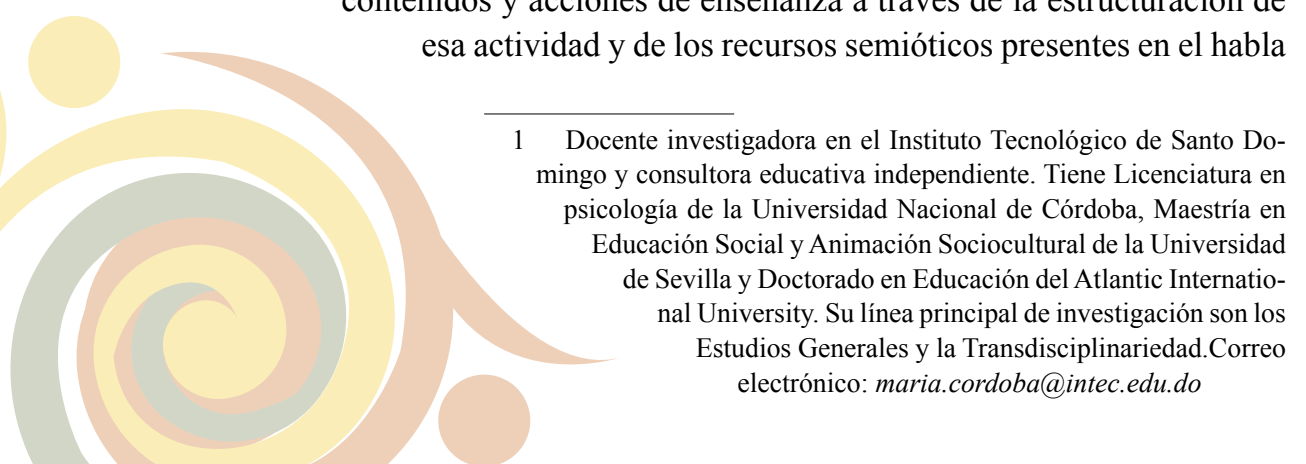


de los involucrados. Por último, se puede establecer un paralelismo entre el constructivismo sociocultural lingüístico y los Estudios Generales, en tanto se asuma una postura que supere la concepción de aprendizaje desde las disciplinas de manera independiente, a la vez que se priorice el vehículo con el que se construye conocimiento que es el lenguaje, además de otras similitudes que llevan a considerarla digna de ser tomada en cuenta como una teoría de soporte para la práctica docente en Estudios Generales.

Palabras clave: Constructivismo sociocultural lingüístico; Estudios Generales; recursos semióticos; relación horizontal; lenguaje.

\begin{abstract}
This article aims to highlight and analyze some characteristics of constructivism and General Studies to build conceptual bridges and methodological encounters between them. It also aims to generate reflection that allows identifying, specifically, the benefits of linguistic, sociocultural constructivism as an appropriate theoretical foundation for General Studies epistemology, whose purpose is to develop ethical citizens, with critical thinking, and sense of the common good, among other characteristics. A horizontal relationship that General Studies proposed and a student-teacher bond, which allows recognizing oneself and others as persons, cannot be founded upon any pedagogical theory. Therefore, it is possible to create bonds with this form of constructivism, in which the process of teaching and learning implies joint activities between students and teachers and occurs around contents and teaching actions through the structuring of those activities and the semiotic resources present in the speech of people involved. Finally, a parallel can be established between linguistic, sociocultural constructivism and General Studies, as long as a position is adopted for the conception of learning to be superseded from disciplines independently, prioritizing, at the same time, the means with which knowledge is built, i.e., language, in addition to other similarities that lead to considering that position to be worthy of being taken into account as a founding theory for teaching practice in General Studies.
\end{abstract}

Keywords: Linguistic sociocultural constructivism; General Studies; semiotic resources; horizontal relationship; language. 


\section{Introducción}

Libres son quienes crean, no quienes copian, y libres son quienes piensan, no quienes obedecen. Enseñar es enseñar a dudar.

Eduardo Galeano

El intento de dar una respuesta a la consulta de un colega de la Universidad de Costa Rica y continuar preguntándome cuál es mi función en relación con los Estudios Generales derivó en un esfuerzo por buscar la teoría pedagógica que mejor acompañara el proceso de enseñanza aprendizaje en los Estudios Generales. En ese momento mi contestación estaba relacionada con el hecho de que, si bien la educación presenta marcos explicativos y avances importantes para el proceso de enseñanza aprendizaje, existen diversas propuestas conceptuales y epistemológicas con aportes interesantes desde algunos tipos de constructivismo, que considero favorecen el proceso académico de los Estudios Generales.

Cesar Coll, quien tanto ha contribuido en el campo educativo, advertía ya desde finales del siglo pasado sobre lo siguiente

El riesgo del eclecticismo que comporta la utilización de los principios constructivistas al margen de las teorías del desarrollo y del aprendizaje en cuyo contexto han sido formulados, ... sobre la tentación del dogmatismo que puede derivarse de la utilización de un único marco teórico de referencia y sobre el carácter excesivamente genérico de principios constructivistas. (1996, p. 154)

Por tanto, no es intención de esta propuesta asumir una teoría como la respuesta única de soporte pedagógico, sino, más bien, revisar cuál es la que mejor acompaña a los Estudios Generales para aprovechar sus postulados y métodos en el accionar académico, apoyado de los marcos de referencia que resulten de utilidad.

El propósito en esta oportunidad consiste en compartir algunas de las características del constructivismo sociocultural lingüístico y reflexionar sobre sus bondades para el proceso enseñanza aprendizaje en los Estudios Generales.

Estoy convencido de que hay que seguir trabajando por lo que queremos, en lo que nos corresponde a todos. Creo que eso es la vida, es construir esperanza, abrir horizontes, tender puentes hacia un futuro mejor. Sembrar alegría y construir esperanza invocando nuestras utopías y trabajando 
tenazmente por realizarlas hasta el último día de nuestra vida. (Latapí, en Rueda, 2009, p. 4)

En una de sus últimas intervenciones en público, Pablo Latapí expresaba, una vez más, su compromiso inquebrantable con la función de la educación como elemento indispensable para construir un mundo mejor. Desde esa postura profundamente humana y pedagógica, resulta, en este caso, como una invitación a preservar los principios de los Estudios Generales y reforzarlos con teorías pedagógicas que resulten soporte y ayuda para la reflexión y la mejora.

\section{El constructivismo}

Sin pretender hacer un análisis exhaustivo, revisemos algunos aspectos generales sobre constructivismo, con la intención de recuperar las características de la modalidad sociocultural lingüística que, según la hipótesis que propongo, reúne varias características idóneas para acompañar el proceso educativo de los Estudios Generales.

De acuerdo con Edwards (1997) y Potter (1998) en Serrano y Pons (2011), cualquier tipo de clasificación de los diversos tipos de constructivismos recoge, explícita o implícitamente, la existencia de tres visiones:

a) Un constructivismo cognitivo que hunde sus raíces en la psicología y la epistemología genética de Piaget, b) un constructivismo de orientación socio-cultural (constructivismo social, socio-constructivismo o co-constructivismo) inspirado en las ideas y planteamientos vygotskyanos y c) un constructivismo vinculado al construccionismo social de Berger y Luckmann (2001) y a los enfoques posmodernos en psicología que sitúan el conocimiento en las prácticas discursivas. (p. 3)

El constructivismo constituye una perspectiva que en cualquiera de sus modalidades intenta explicar la naturaleza humana y los fenómenos relacionados con la construcción de conocimiento y la interpretación de la "realidad", desde una posición epistemológica, psicológica y educativa. En una de sus variantes más avanzadas, sostiene que el conocimiento se forma a través de la relación con las experiencias, acciones o procesos de construcción en un contexto social. De este modo, lo que se conoce ocurre por la interacción del individuo con otras personas y con el medio a través de la mediación del lenguaje. 
Damasio (2010) hace referencia al continuum cuerpo-cerebro-mente que aparece estrechamente relacionado en cualquier proceso educativo, sin ser reducido el uno al otro, y cuyas conexiones no serían posibles sin la mediación del lenguaje.

Por su parte, el planteamiento de Vygotsky plantea:

Son la sociedad y la cultura la razón de los razonamientos y las decisiones. En esta forma, el principal propósito de la educación es cultivar en los estudiantes un repertorio de estrategias de comportamiento y de conocimiento, flexibles y creativas, que les permita reconocer la complejidad de las situaciones y contar con alternativas de solución. (Valencia, 2016, p. 79)

La idea original del constructivismo es que el conocimiento y el aprendizaje ocurren como resultado de una dinámica en la que el sujeto juega un papel activo, y el objeto es conocido según los marcos interpretativos de este sujeto, de tal forma que el conocimiento y el aprendizaje son parte de la actividad mental constructiva, de acuerdo con la cual las personas interpretan la experiencia. Rosario Cubero (2005) lo concibe desde una epistemología constructivista y una ontología realista (Valencia 2016).

Coll (2001), en Serrano y Pons (2011), plantea que las representaciones individuales y las actividades sociales culturalmente organizadas mantienen una interconexión en la cual se influyen mutuamente y generan una relación fluida y transaccional, de modo que para entender el proceso de construcción de conocimiento estudiantil es necesario que ambas sean tomadas en cuenta. Para este autor, el aprendizaje se trata de un proceso externo e interno que tiene lugar en el contexto donde ambos aspectos se relacionan y se afectan mutuamente.

Nuthal, 1997 y Salomón, 2001, ambos en Serrano y Pons (2011, p. 9) indican que si incorporamos las perspectivas sociocultural y lingüística al modelo cognitivo de los procesos mentales, es posible vislumbrar cómo el lenguaje y los procesos sociales del aula constituyen las vías a través de las cuales los estudiantes adquieren y retienen el conocimiento, fundamentalmente porque resulta muy útil considerar los procesos mentales como una propiedad de los individuos que actúan en entornos organizados culturalmente.

\subsection{Constructivismo cognitivo}

El constructivismo cognitivo de Piaget (1964) considera que el conocimiento surge de la actividad adaptativa que se da en la mente de las personas. Piaget 
planteaba que el aprendizaje ocurre cuando se relacionan representaciones nuevas con las que la persona ya traía previamente y que en la mente es donde están almacenadas las representaciones mentales, esquemas y modelos que resultan la base en la que se ensamblan las representaciones nuevas.

Partiendo de esta posición, se entiende que el proceso cognitivo se desarrolla a través de la acción de mecanismos como la asimilación y acomodación. La asimilación se refiere a la incorporación de información a los esquemas existentes en el sujeto; la acomodación a la transformación de esos esquemas a nuevas situaciones y experiencias. La puesta en marcha de estos mecanismos constituye un proceso continuo de equilibración. Piaget concibió un proceso de desarrollo a través de la construcción personal apoyada en conocimientos previos que comprende la reorganización cognitiva de los esquemas y estructuras internas autorreguladoras del sujeto. En cierto sentido, su postura da predominancia a los procesos internos del individuo para lograr aprendizajes.

\subsection{Construccionismo social}

El construccionismo social es una variante del constructivismo compartida por enfoques lingüísticos y sociolingüísticos de la cognición y el aprendizaje. En esta perspectiva, el conocimiento se construye de manera social dentro de un contexto, se sitúa en la interacción entre la mente y el exterior del individuo, a través del lenguaje y en acciones ubicadas, organizadas y coconstruidas en la interacción social.

Apoyos del construccionismo social:

La convicción de que las personas, los objetos y los hechos existen en un mundo lingüístico, en el que el lenguaje sirve para representar, describir la realidad y comunicar estados mentales, desde esta concepción el lenguaje no es una "vía" o un canal por el que se transmiten mensajes, sino una actividad en la que se produce el significado. En esta forma, el discurso no consiste en una representación del pensamiento en el lenguaje, sino un modo social de pensar. (Mercer, 1996 en Valencia, 2016, p. 25)

\subsection{Constructivismo sociocultural y lingüístico}

El constructivismo sociocultural surge de la mano de Vygotsky (1989a; 1995) y plantea que los procesos psicológicos superiores ocurren a partir de relaciones dialécticas de las personas con el medio, como una aproximación sociocultural de 
lo humano. Lo que sugiere que el individuo construye significados actuando en un entorno estructurado e interactuando con otras personas de forma intencional (Serrano y Pons, 2011).

Wertsch $(1985 ; 1991)$, quien analiza la obra de Vygotsky, observa que en los planteamientos de este último se encuentran tres tesis que hacen aportes decisivos a la perspectiva constructivista sociocultural y lingüística:

- Método genético o evolutivo: Plantea que el estudio del desarrollo de los procesos psicológicos debe hacerse a través del análisis de su evolución con el fin de entender lo que significa. Es necesario partir de la acción humana que implica cambio, a su vez comprender que la acción humana implica analizar su propio desarrollo, sus orígenes y sus transformaciones genéticas.

- Doble origen social del funcionamiento mental: Considera la vida social, las interacciones entre las personas y las actividades culturales como los factores que dan origen a los procesos psicológicos superiores. "Los procesos psicológicos superiores adoptan tanto la dimensión individual como social, pasando de lo interpsicológico a lo intrapsicológico, en lo cual la dimensión social es anterior en el tiempo, y la dimensión individual se deriva de ella" (Vygotsky, 1979b, en Valencia, 2016). De esta manera el proceso de construcción del conocimiento surge de la interdependencia entre lo individual y lo social. Para explicar esta relación de interdependencia, Vygotsky utilizó los conceptos de interiorización, zona de desarrollo próximo y apropiación. La interiorización es el proceso en el que ciertos elementos de la actividad realizada por el individuo en el plano externo pasan a ejecutarse en el plano interno. La interiorización fue concebida por Vygotsky como un proceso en el que ciertos aspectos de la estructura de la actividad que se han realizado en el plano externo pasan a ejecutarse en un plano interno, en un proceso intraindividual. El proceso de interiorización de contenidos y herramientas psicológicas de la cultura permiten el tránsito de lo interpersonal a lo intrapersonal, y desde allí surge el concepto de zona de desarrollo próximo.

La zona de desarrollo próximo es:

- La distancia entre el nivel real de desarrollo, determinado por la capacidad de resolver independientemente un problema, y el nivel de desarrollo potencial, determinado a través de la resolución de un problema bajo la guía de un adulto o en colaboración con otro compañero más capaz. (Vygotsky, 1989a, p. 133)

- La apropiación se refiere a como el individuo hace suyos los productos de la cultura, a través de ésta el individuo "busca la reconstrucción de facultades y 
modos de comportamiento desarrollados históricamente, actuando a partir de opciones semióticas que le permiten reflejar su identidad" (Valencia, 2016).

- Mediación semiótica: Los procesos de mediación semiótica conciben la actividad humana como un fenómeno mediado por signos y herramientas. El ser humano ha utilizado materiales y herramientas psicológicas, basadas en sistemas de signos, para transformar activamente su entorno físico. Este sistema de signos se produce y comparte de manera social e incide en procesos como el habla, el pensamiento y la acción. De esta manera, el lenguaje constituye el sistema de signos preferido para el desarrollo psicológico humano. Éste promueve la mediación de las relaciones de la persona consigo misma a través de diálogos internos (pensamientos) y con los demás individuos.

Como respuesta a la ausencia de una explicación general que permita comprender los elementos que intervienen y modulan los procesos de enseñanza y aprendizaje escolar, surge la perspectiva constructivista sociocultural y lingüística. La dicotomía marcada por el constructivismo cognitivo (orientado a lo individual/interno) y el construccionismo social (orientado a lo social/externo) trazan la frontera entre el pensamiento y el lenguaje. La perspectiva sociocultural lingüística intenta acercar estas dos visiones. Busca integrar el lenguaje y los procesos sociales en el aula para lograr comprender los procesos escolares de enseñanza y aprendizaje.

Algunos estudios sugieren que la incorporación del enfoque sociocultural y lingüístico con la propuesta constructivista cognitiva de los procesos mentales permite ver cómo estudiantes adquieren y retienen conocimiento a través del lenguaje y los procesos sociales. Esto sugiere que la educación es un proceso comunicativo que tiene lugar gracias a las actividades realizadas conjuntamente entre docentes y estudiantes, mediadas por el lenguaje.

Según Coll (2001), este enfoque incorpora planteamientos socioculturales y lingüísticos del constructivismo cognitivo, en los que "la construcción individual del conocimiento que llevan a cabo los estudiantes está inmersa y es inseparable de la construcción colectiva que llevan a cabo profesores y estudiantes en este entorno específico y culturalmente organizado que es el aula".

En fin, el aprendizaje es considerado un estímulo que activa diversos procesos mentales, los cuales se desenvuelven en el marco de interacción con otras personas en diversos contextos y siempre a través del lenguaje. Esos procesos mentales, de cierto modo, reproducen esas formas de interacción social "internalizadas en el proceso de aprendizaje social hasta convertirse en modos de autorregulación" (Carrera y Mazzarella, 2001). 


\subsection{Constructivismo en la enseñanza y aprendizaje}

La psicología de la educación en la actualidad está influenciada por la visión constructivista del psiquismo humano. Es común usar como referencia los principios constructivistas del funcionamiento psicológico para comprender y explicar mejor los procesos de desarrollo y de aprendizaje, y los procesos educativos. Además, frecuentemente se parte de estos principios para elaborar y fundamentar propuestas con el fin de innovar y mejorar la educación (Coll, 1990).

Desde los enfoques constructivistas se busca entrelazar los procesos de enseñanza y aprendizaje, que han estado separados tradicionalmente desde las concepciones de la psicología de la educación. El constructivismo considera imposible entender cómo se aprende, si se desvincula de cómo se desarrolla el proceso de enseñanza y viceversa; por esta razón, en las últimas décadas se ha revalorizado el proceso de enseñanza y aprendizaje de forma integrada. Para Vygotsky y la perspectiva socioconstructivista, "el aprendizaje escolar es un fenómeno social, construido por cada estudiante durante la actividad que se desarrolla en colectividades de aprendices, y que está conectada con lo histórico y lo social" (Valencia, 2016).

Para Coll, Mauri y Onrubia (2008), el aprendizaje en contextos educativos es "un proceso de construcción y reconstrucción de significados y atribución progresiva de sentido, llevado a cabo por el alumno o aprendiz y referido a contenidos complejos culturalmente elaborados, establecidos y organizados".

Valencia (2016) agrega:

La concepción constructivista de la enseñanza y el aprendizaje escolar, como heredera del modelo Vygotskyano, introduce la noción de atribución de sentido para decir que cuando los estudiantes aprenden, no solo alcanzan una comprensión de lo que se aprende, sino que hacen suyo ese conocimiento como forma de ver la realidad. De esta forma, el aprendizaje, además de modificar la comprensión sobre las cosas, transforma el sentido que estas tienen para el estudiante. Por tanto, la atribución de sentido al aprendizaje se propicia de acuerdo con un conjunto de experiencias emocionales que provoca una situación de aprendizaje. Gracias a este proceso es posible relacionar lo que aprendemos con componentes motivacionales, afectivos y relacionales de los aportes del estudiante al acto de aprender. (p. 40)

Desde el enfoque constructivista, la atribución de sentido al aprendizaje y la construcción de significados deben visualizarse desde una óptica sociocultural desarrollada en un contexto de comunicación interpersonal que va más allá de la 
dinámica interna de los procesos de pensamientos del estudiantado. El proceso de enseñanza y aprendizaje sucede dentro del marco de actividad conjunta entre estudiantes y docentes, alrededor de contenidos y acciones de enseñanza a través de la estructuración de esa actividad y de los recursos semióticos presentes en el habla de los sujetos involucrados.

La participación dentro del contexto social propicia oportunidad al individuo de iniciar la interacción verbal que permite el surgimiento de procesos mentales avanzados, es esta interacción social la que adentra a la persona en nuevas formas de actividades mentales, las cuales, más adelante, interioriza y convierte en acciones para solucionar problemas en un contexto independiente (Gutiérrez, 2004).

Por otro lado, el aprendizaje y la comunicación de conceptos están directamente ligados al pensamiento verbal; esto significa que los contenidos de una disciplina están enlazados con las formas verbales que los expresan. Los contenidos están constituidos por palabras, por lo tanto, el aprendizaje de éstos señala el desarrollo de operaciones psicológicas de orden superior. Al respecto, Gutiérrez (2004) plantea:

El desarrollo del intelecto y la adquisición de los conceptos científicos están mediados por la interacción social, el diálogo calificado entre la persona competente y quien aprende, permite la evolución y maduración de los procesos mentales superiores, en la medida que las funciones que se comparten lingüísticamente en el plano interpsicológico (interpersonal) son interiorizadas por el sujeto, usadas para atender a demandas del medio ambiente y regular la conducta. (p. 137)

\section{Estudios Generales}

Algunas apreciaciones sobre los Estudios Generales permitirán observar las bondades de la teoría pedagógica mencionada. Si bien contamos con basta y rica información sobre los Estudios Generales, en este espacio nos limitamos a algunos aspectos que considero indispensables en el proceso académico y lo primero que resalto es que los Estudios Generales representan una propuesta que atiende las demandas de la sociedad de hoy y la complejidad e incertidumbre del mundo actual. Así también, somos conscientes de que resulta indispensable que, desde la construcción del currículo, se considere que los programas universitarios provean herramientas que posibiliten a sus estudiantes comunicarse en un ambiente multi e interdisciplinario. Por su parte, en el ámbito profesional docente asistimos a propuestas que, a veces, son modas; otras impuestas como camisas de fuerza y 
que permean con enfoques y equipos profesionales diversos los espacios académicos, confundiendo, con frecuencia, a docentes que viven en una carrera por cumplir programas, normas y reglas, además del agobio por el pluriempleo. Sin embargo, los Estudios Generales con su visión integral del conocimiento, ofrecen un enfoque de respeto democrático, de invitación al trabajo en equipo y modalidad horizontal en el hecho educativo.

Ahora bien, para poder compenetrarse y trabajar en conjunto, se necesita de un "lenguaje y una formación básica que nos permita enriquecer nuestra perspectiva disciplinaria con los aportes de las otras disciplinas" (Tubino, 2011) resulta en esa modalidad de trabajo que contribuye a la formación integral que proveen los Estudios Generales. Esta superación de las fronteras disciplinares para acercarse al objeto de conocimiento no pretende negar las disciplinas o eliminarlas, sino más bien entender sus limitaciones para la comprensión integral de la problemática de estudio y favorecer la relación entre éstas en el proceso de enseñanza aprendizaje, de manera que se asemeje al mundo real, donde todas las áreas disciplinarias se encuentran conectadas. La educación universitaria con los Estudios Generales en su currículo busca superar las barreras artificiales que se han impuesto entre los saberes y reconocer el valor disciplinar que se establece en forma didáctica para profundización y especialización.

Los Estudios Generales, además -y tal vez, sobre todo- promueven activa y deliberadamente las conexiones entre las diversas áreas del saber, propiciando la reconstrucción de su tejido (complexus), así como el entendimiento de las razones históricas de su parcelación (disciplinarización) y los efectos que esto ha tenido y tiene en los procesos de aprendizaje y producción cultural. Además de caracterizarse por propiciar la conexión e integración de saberes provenientes de los diversos campos del conocimiento (ciencias sociales, ciencias naturales y humanidades), los Estudios Generales incorporan integralmente las experiencias y vivencias cotidianas de los estudiantes en los procesos de aprendizaje. (Dewey, 2004 en Córdoba y Vélez, 2017, p. 42)

Diversos autores han hecho aportes desde perspectivas diferentes de los Estudios Generales, que evidencian cada vez más la imperiosa necesidad de incorporarlos en el currículo como elemento fundamental en la formación de una ciudadanía capaz de enfrentar la incertidumbre y complejidad del mundo actual. Al ser aportes diferentes entre sí, posibilitaron desplegar un abanico de posibilidades que permitió revisar, si el enfoque pedagógico seleccionado resulta acorde para acompañar el proceso de enseñanza aprendizaje de los Estudios Generales. 
El propósito de esta presentación como se dijo es revisar las características del constructivismo sociocultural lingüístico y su pertinencia como enfoque pedagógico para los Estudios Generales, por lo que se mencionan solo algunas referencias para contextualizar.

Las aptitudes cognitivas humanas solo pueden desarrollarse en el seno de una cultura que ha producido, conservado y transmitido un lenguaje, una lógica, un conjunto de saberes y de criterios de verdad. ... [los Estudios Generales] incitan y promueven deliberadamente la contextualización de la información o idea, adentrándose el tejido del que está compuesta, en lugar de pretender parcelarla y compartamentalizarla, como hacen las disciplinas. (Morin en Vélez, 2011, p. 16)

Luis E. González comprende la formación general como "un conjunto coherente de conocimientos, destrezas y habilidades; y valores que constituyen una base cultural sólida, sobre la cual los estudiantes pueden construir su especialización profesional, recibiendo así una formación más integral" (González en Castro, Colpas, Barnett, Roa, Cabrera y Tomás del Valle, et al., 2018).

Más adelante Rodríguez (2011) cita a Claudio Prieto en su definición de Estudios Generales:

Lo característico de la educación general es su enfoque del contenido como proceso antes que, como conclusión, su acercamiento a los reclamos de validez con sano escepticismo y con entendimiento del contexto histórico en que estos se dan: en otras palabras, la visión del conocimiento como proceso de creación humana en todos los contextos -literario, filosófico, científico ... el examen de hipótesis alternas, el entendimiento del proceso de creación, el análisis de las premisas del razonamiento del autor-, éstos son todos elementos del acercamiento al conocimiento como estructura del pensamiento y como cultura que, a mi entender, es de la esencia misma de la educación general. (p. 36)

Alberto Roa Valero menciona la posición de la Pontificia Universidad Católica del Perú (PUCP) y presenta los Estudios Generales como el medio por el que los estudiantes adquieren conocimientos y habilidades que los acompañarán en el transcurso de su vida universitaria y profesional. Esto, con el fin de que sea un profesional exitoso y también una "persona de cultura amplia, académicamente sólida, con valores éticos y con una mirada responsable hacia la Sociedad" (Castro et al., 2018). 
Algunas características de los Estudios Generales:

- Comprende al ser humano en su unidad integrada (consigo mismo, con otros seres humanos y con su entorno).

- Comprende el conocimiento en su unidad epistémica.

- Comprende la realidad en su complejidad y unidad indisoluble.

- Articula esa triple comprensión por medio de la transdisciplinariedad (Vélez, 2011).

Luis E. González (en Uninorte, 2018) agrega características:

- Desarrolla en el estudiante una visión crítica y actualizada de la cultura.

- Desarrolla en el estudiante una actitud positiva hacia el conocimiento interdisciplinario, en función de la adquisición de una personalidad intelectual analítica.

- Desarrolla en el estudiante el manejo de enfoques conceptuales y metodológicos de las ciencias, en la perspectiva de inculcar un desarrollo profesional con sentido social.

- Desarrollar las capacidades analíticas y reflexivas de los educandos, procura la autorrealización, genera una mayor sensibilización, y motiva tanto la relación con la comprensión de la realidad social, como con el entorno cultural.

- Fomenta la adquisición de capacidades para la autogestión y el autodesarrollo (Guedez, 1991, en Uninorte, 2018).

Además, Vélez (2011, p. 16), agrega:

La educación general reconoce la porosidad y vinculación de los saberes, así como la insuficiencia y limitación que representa la manera en que han sido organizados en las universidades (disciplinas, departamentos, facultades, carreras, etc.). De ahí que su principal aportación a la formación integral de los y las estudiantes provienen de su énfasis en develar los fundamentos epistemológicos, económicos, políticos, en fin, culturales en sentido amplio, de todas las maneras de producir y organizar el conocimiento, tanto a través de la historia como en la actualidad; y la indisoluble vinculación de estos procesos con nuestra realidad y con las formas en que la representamos, construimos y reconstruimos, pero sobre todo experimentamos, disfrutamos, sufrimos y vivimos.

Los Estudios Generales son una realidad en el ámbito académico universitario desde hace ya varias décadas. A través del tiempo se han hecho esfuerzos por mejorar su calidad y porque la formación general permee el currículo de todos los perfiles profesionales de la oferta académica de las instituciones de educación superior. 
Cada vez hay mayor compromiso y consciencia por parte de las instituciones de educación superior por alcanzar la formación integral que el profesional de hoy necesita. Esta es una tarea que debe considerarse continua, no basta con incluir en los documentos de la universidad la propuesta de Estudios Generales, sino que debe mantenerse actualizada y debe dársele vida constante en las aulas, por ser una propuesta dinámica, en constante cambio, de acuerdo con el contexto y los involucrados en el proceso y la relación entre ellos. La programación de las asignaturas de la educación general no es por esto menos rigurosa que cualquier otra, y es la modalidad adecuada para garantizar la calidad del perfil profesional esperado.

Desde la perspectiva de los Estudios Generales, el estudiante es "un ser humano que se inserta en un contexto social y natural que a la vez que lo condiciona es condicionado por él, más que en un cliente que hay que complacer o una mercancía a la que hay que añadirle valor" (Vélez, 2014). Por esto los aprendizajes generados a través de los Estudios Generales tienen un carácter comunitario, son aprendizajes que mantienen al estudiante conectado con los demás y con su entorno; esa conexión permite que el individuo comprenda, a su vez, la relación que hay entre los saberes y las experiencias que forman parte de una totalidad, porque, al mismo tiempo, analiza la realidad no parcelada en una forma natural de análisis: el pensamiento crítico.

\section{Conclusión}

Cuando se hace un recorrido por las concepciones básicas del constructivismo sociocultural lingüístico y los Estudios Generales resulta evidente la afinidad entre ambos. La perspectiva del constructivismo sociocultural lingüístico apela a una explicación del aprendizaje que parte de la interacción del mundo interno y externo de la persona, cuyo vínculo de realización para esa interacción entre pares, contexto y personas de otros contextos es el lenguaje. De esta forma se activan las capacidades cognitivas, socio cognitivas y metacognitivas, es decir, que supone un método activo de enseñanza y holístico de aprendizaje. La modalidad dinámica e integradora de saberes, así como la formación integral de los estudiantes que caracteriza los Estudios Generales, puede asumir este tipo de constructivismo como favorable para su práctica pedagógica, por su postura conceptual y epistemológica que encaja perfectamente con sus postulados básicos. 
Mediante el uso del lenguaje "las personas podemos representarnos nuestro propio conocimiento y dar sentido a nuestra experiencia y nuestra actividad y al mismo tiempo compartir esos conocimientos y experiencias con otros" (Coll y Onrubia, 2001, p. 22). Estos procesos de construcción del conocimiento entre docentes y estudiantes acontecen en un clima de horizontalidad democrática utilizando estrategias discursivas y recursos semióticos a los que docentes y estudiantes recurren, de manera habitual, en su intento de establecer sistemas de significados compartidos cada vez más ricos y complejos, en torno a las actividades y contenidos del conocimiento (Coll y Onrubia, 2001).

Elementos tan afines desde esta corriente pedagógica con los Estudios Generales resultan interesantes como para explorar las ventajas que aportaría asumirla.

La función tanto representativa como comunicativa del lenguaje lo convierte en un instrumento para pensar y aprender de los otros y con los otros, para compartir con otros nuestros conocimientos y experiencias, deseos, intereses, motivaciones, sueños, y contrastarlos con los de los demás, "representarlos de diferentes maneras, negociarlos y eventualmente modificarlos, como resultado del contraste y la negociación" (Coll y Onrubia, 2001).

Gracias al lenguaje y su enorme potencial como instrumento semiótico, su capacidad para crear transformar y comunicar significados, es posible que docentes y estudiantes organicen su actividad de manera conjunta y coconstruyan conocimientos, lo cual evidentemente favorece la postura integrativa que promueven los Estudios Generales.

En el constructivismo sociocultural lingüístico se da importancia tanto al individuo y sus procesos internos como al medio y las demás personas con las que interactúa para que pueda ocurrir la adquisición de conocimientos. Es una propuesta superadora del aprendizaje desde un solo lugar o desde las disciplinas de manera independiente, porque prioriza el vehículo con el que se construye conocimiento, que es el lenguaje, y el lenguaje no es de ninguna manera solo oral o escrito, hay un lenguaje artístico, un lenguaje corporal, un lenguaje gestual, un lenguaje del silencio, etc. La inclusión del arte y la superación de fronteras disciplinares de los Estudios Generales se ven enriquecidas desde esta propuesta pedagógica que valora los mismos principios.

Esta concepción pedagógica supera también la postura de que el aprendizaje acontece en el individuo como un ser pasivo que recibe directamente los conocimientos y los procesa internamente, sino que se trata, más bien, de un individuo 
que construye los conocimientos a través de sus experiencias en su relación con el medio social. Así mismo, los Estudios Generales promueven una formación en la cual el aprendizaje ocurre en contacto con el contexto social, histórico y cultural, mientras el estudiante aprecia los saberes en su estado natural, con sus interrelaciones en el mundo real. Además, los Estudios Generales también consideran al estudiante un ente activo dentro del proceso de aprendizaje, y dan importancia a la experiencia y al significado que tienen los conocimientos para éste.

El lenguaje, la interacción social, la cultura, la integración de saberes, el sentido y la experiencia son todos elementos relevantes para el aprendizaje, tanto en el constructivismo sociocultural lingüístico, que explica cómo se construyen los conocimientos, como en los Estudios Generales que proponen la formación integral del ser humano, y la integración de conocimientos. Por tanto, es posible afirmar que la hipótesis de la que partimos no solo se demuestra, sino que además es superada. Ahora bien, eso ocurre en la teoría, sería deseable que se llevara a cabo al menos una prueba piloto para contrastar estas reflexiones con la práctica.

Estos aspectos aquí analizados sugieren el beneficio de capacitar docentes en esta modalidad constructivista sociocultural lingüístico, a fin de facilitar la labor académica en Estudios Generales y fortalecerla.

\section{Referencias}

Carrera, B. y Mazzarella, C. (2001). Vygotsky: Enfoque sociocultural. Educere, 5(13). Recuperado de: https://www.redalyc.org/pdf/356/35601309.pdf

Castro, A., Colpas, E. (Eds.). (2018). Reflexiones sobre los Estudios Generales en la educación superior uninorte. Universidad del Norte. Recuperado de : http://manglar.uninorte.edu.co/ bitstream/handle/10584/7888/9789587418750\%20eReflexiones\%20 sobre $\% 20$ estudios $\% 20$ en $\% 20$ Educaci $\%$ C 3\%B 3 n $\% 20$ Superior. pdf? sequence $=1 \&$ isAllowed $=\mathrm{y}$

Coll, C. (1996). Constructivismo y educación escolar: Ni hablamos siempre de lo mismo ni lo hacemos siempre desde la misma perspectiva epistemológica. Anuario de Psicología, 69. 154-178. 
Coll, C. (1990). Constructivismo y educación: La concepción constructivista de la enseñanza y el aprendizaje. Psicología de la Educación Escolar (pp. 157-186). Alianza.

Coll, C., y Onrubia, J. (2001). Estrategias discursivas y recursos semióticos en la construcción de sistemas de significados compartidos entre profesor y alumno. Revista Investigación en la Escuela, 45, 21-31.

Coll, C., Mauri, T., y Onrubia, J. (2008). Ayudar a aprender en contextos educativos: El ejercicio de la influencia educativa y el análisis de la enseñanza. Revista de Educación, 346, 33-70.

Córdoba, M., y Vélez, W. (2017). Evaluación del papel de los intercambios de docentes de Estudios Generales para la formación integral del estudiante. Ciencia y Sociedad, 42(2), 39-52.

Damasio, A. (2010). Y el cerebro creó al hombre. Ediciones Destino.

Gutiérrez, M. (2004). Desarrollo cognitivo yeducación. Universidad de Manizales

Rueda, M. (2009). In memoriam: Dr. Pablo Latapí Sarre (1927-2009). Perfiles educativos, 31(125), 3-6. Recuperado de: http://www.scielo.org.mx/scielo. php?script=sci_arttext\&pid=S0185-26982009000300001\&lng=es\&tlng=es.

Piaget, J. (1964). Seis estudios de psicología. Editorial Labor.

Rodríguez, J. (2011). El papel de los Estudios Generales en los estudios universitarios contemporáneos. Estudios, 9(97), 19-38

Serrano, J. M. y Pons, R. M. (2011). El constructivismo hoy: Enfoques constructivistas en educación. Revista Electrónica de Investigación Educativa, 13(1). http://redie.uabc.mx/vol13nol/contenido-serranopons.html

Tubino, F. (2011) La formación humanista para el desarrollo y el papel de los Estudios Generales en la educación universitaria. En Contexto y sentido de los Estudios Generales. Pontificia Universidad Católica del Perú. (pp. 79-108)

Valencia, V. (2016). Dimensión emocional en la atribución de sentido al aprendizaje, en un entorno educativo universitario hibrido [Tesis de posgrado]. Universidad Tecnológica de Pereira. 
Vélez, W. (2011). Una educación general transdisciplinaria para el fortalecimiento de la Universidad. Revista Umbral, 69, 5-32.

Vélez, W. (2014). Pertinencia de los Estudios Generales en los currículos universitarios en el siglo XXI. Seminario Internacional de Estudios Generales.

Vygotsky, L. (1989a). El desarrollo de los procesos psicológicos superiores. Critica.

Vygotsky, L. (1995). Pensamiento y Lenguaje. Paidós.

Wertsch, J. (1985). Introduction. En Wertsch, J. (1985). Culture, communication and cognition: Vygotskyan perspectives. Cambridge University Press.

Wertsch, J. (1991). Voces de la mente. Visor. 